\title{
COVID-19 in older adults: clinical, psychosocial, and public health considerations
}

\author{
John P. Mills, ${ }^{1}$ Keith S. Kaye, ${ }^{1}$ and Lona Mody ${ }^{2,3}$ \\ 1Department of Internal Medicine, Division of Infectious Diseases, and ²Department of Internal Medicine, Division of \\ Geriatric and Palliative Medicine, University of Michigan Medical School, Ann Arbor, Michigan, USA. ${ }^{3}$ Geriatrics Research \\ Education and Clinical Center, Veterans Affairs Ann Arbor Healthcare System, Ann Arbor, Michigan, USA.
}

\section{Epidemiology of COVID-19 in older adults}

In December 2019, a new severe respiratory illness emerged in Wuhan, China and was linked to a novel beta-coronavirus that likely originated from zoonotic transmission (1). As of April 16, 2020, the severe acute respiratory syndrome coronavirus-2 (SARS-CoV-2) has caused over 2 million confirmed infections and 138,000 deaths worldwide (2). The rapid spread of SARS-CoV-2 has resulted in unprecedented stress on health care systems across the globe. Complications of coronavirus disease 2019 (COVID-19, the name of the infection caused by SARS-CoV-2) have been particularly severe among older adults, who are the focus of this Viewpoint. Existing literature suggests that age is an important predictor of poor outcomes among patients with COVID-19. Multiple case series have found escalating rates of severe disease and mortality with increases in age. Data from 44,672 confirmed COVID-19 cases in China revealed an overall case-fatality rate (CFR) of $2.3 \%$, which rose to $8.0 \%$ among patients $70-79$ years old and to $14.8 \%$ for those 80 years and older (3). Similar trends have been identified in early data from the United States. The Morbidity and Mortality Weekly Report (MMWR) revealed 2,449 COVID-19 cases between February 12, 2020 and March 16, 2020 and an intensive care unit (ICU) admission rate of $8.1 \%-18.8 \%$ and CFR of $2.7 \%-4.9 \%$ for adults $65-74$ years of age (4). The ICU admission rate rose to $10.5 \%-31.0 \%$, with a $4.3 \%-10.5 \%$ CFR, for those $75-84$ years of age, and for patients 85 years and older, an ICU admission rate of $6.3 \%-29.0 \%$, with a $10.4 \%-27.3 \%$ CFR, was reported.

Early data also suggest worse outcomes for men compared with women. A large case series in China found that 459 of 1096 infections (41.9\%) occurred in women (5). Of 67 patients that met the composite endpoint of ICU admission, mechanical ventilation, or death, only 22 (32.8\%) were women. However, patient selection for this study was not random; therefore, the possibility of reporting bias could not be excluded. Data from Italy and Korea have both indicated higher CFRs among men compared with women $(6,7)$. It is unclear if there is a biological cause for this sex-specific disparity or if these differences are purely a consequence of a higher prevalence of comorbid conditions, occupational exposure, and smoking among men. Sex differences in outcomes may be attenuated among older adults. In a cohort of 788 patients from Zhejiang Province, China, there was a higher frequency of women among infected patients 60 years or older versus those younger than 60 years (57.4\% vs. $46.5 \%, P=0.021)(8)$.

Unsurprisingly, higher rates of severe COVID-19 are reported among patients with comorbid chronic medical conditions. Centers for Disease Control and Prevention (CDC) data on 7,162 laboratory-confirmed COVID-19 cases in the United States identified at least one chronic medical condition in 2,692 cases (37.6\%) (4). The most common medical conditions were diabetes mellitus (10.9\%), chronic lung disease $(9.2 \%)$, cardiovascular disease $(9.0 \%)$, immunocompromise $(3.7 \%)$, and chronic renal disease (3.0\%). The proportion of patients with at least one chronic condition was much higher among hospitalized patients (71\%) and ICU-admitted patients (78\%) compared with those who did not require hospitalization (27\%). The hospitalization rate for patients 65 years of age or older with at least one chronic medical condition was $41.7 \%-44.5 \%$, compared with $16.8 \%-18.3 \%$ for those without any underlying condition. We anticipate

Conflict of interest: The authors have declared that no conflict of interest exists.

Copyright: () 2020, American Society for Clinical Investigation.

Reference information: /CI Insight. 2020;5(10):e139292. https://doi. org/10.1172/jii.insight.139292. learning more about the epidemiology and pathophysiology of COVID-19 in the months to come.

\section{Clinical considerations in caring for older adults with COVID-19}

COVID-19 is a respiratory tract illness that commonly causes fever, cough, fatigue, shortness of breath, myalgias, sore throat, headache, and chills $(5,9,10)$. Nasal congestion and diarrhea also occur infrequently. Complaints of shortness of breath or anorexia were more common among patients requiring ICU admission $(P<0.001)(9)$. Shortness of breath also appears to be a more common complaint among older adults 
( $12 \%$ for patients $>60$ years vs. $3 \%$ for patients $<60$ years) (8). Although $89 \%$ of patients experienced a fever (defined as a temperature greater than $99.5^{\circ} \mathrm{F}$ ) during their hospitalization in one study, only $44 \%$ were febrile at the time of admission (9).

Though symptoms generally are more pronounced in older adults, asymptomatic or presymptomatic infections have been described in this population. During a point-prevalence survey conducted as part of an outbreak investigation at a nursing home in King County, Washington, 13 of $23(57 \%)$ residents who tested positive were asymptomatic at the time of testing. Ten of 13 of these patients $(77 \%)$ subsequently developed typical COVID-19 symptoms upon reevaluation 7 days after initial testing (11).

Common laboratory abnormalities include lymphopenia and thrombocytopenia (5). Higher rates of lymphopenia and anemia, lower albumin, and higher aspartate transaminase (AST) and C-reactive protein (CRP) are found in older adults compared with younger patients (8). Chest computed tomography (CT) is more sensitive than chest $\mathrm{x}$-ray for detection of pulmonary disease. Bilateral pulmonary infiltrates that can consist of ground glass opacifications, consolidations, or both are among commonly found manifestations (10).

The incubation period for COVID-19 is typically 4-6 days, with $98 \%$ of patients developing symptoms within 11 days of exposure (12). The median time from the development of first symptoms to dyspnea was 5 days and time to hospital admission was 7 days (9). Symptomatic older adults are more likely to require ICU admission ( $9.6 \%$ vs. $1.4 \%, P<0.001)$ and develop acute respiratory distress syndrome (ARDS) $(16.9 \%$ vs. $5.4 \%, P<0.001)$ compared with patients less than 60 years of age (8). Incidence of concomitant thromboembolism is high among critically ill COVID-19 patients (13). Outcomes can be devastating in the setting of sustained nursing home transmission. One Seattle area outbreak of 101 residents resulted in a CFR of $33.7 \%$ (14). This outbreak highlighted infection prevention disparities, deficiencies, and staffing challenges within a sector that cares for one of our most fragile populations.

There is no clearly established effective treatment for COVID-19 at this time. Care is predominantly supportive, including antipyretics and supplemental oxygen, as well as mechanical ventilation for those with respiratory failure. No specific therapies are recommended by the Infectious Disease Society of America outside of a clinical trial (15). Hydroxychloroquine, an oral antiparasitic agent, has been used widely with a paucity of supporting data. Ongoing promising clinical trials include those to assess remdesivir, an intravenous RNA polymerase inhibitor, and tociluzimab, an IL-6 inhibitor. Because SARS-CoV-2 binds to the angiotensin-converting enzyme 2 (ACE2) receptor, some have postulated that use of ACE inhibitors and angiotensin receptor blockers, which increase ACE2 expression, could worsen COVID-19 outcomes (16). However, there are no clinical data to support this hypothesis and concern exists regarding abrupt discontinuation, particularly among older adults at risk for cardiovascular events. The American Heart Association recommends continuing these medications in patients with COVID-19 unless advised otherwise by their physician (17).

\section{Psychosocial considerations}

With community spread increasing, health care facilities have been urged to suspend all nonessential visits. This guidance has been beneficial in delaying or reducing nosocomial spread within hospitals, assisted living facilities, and nursing homes. However, unintended consequences of social distancing have resulted in more isolation among older nursing home residents, reduced physical activity, as residents are unable to use rehabilitation gyms, and lack of friendly visitors as a way to enhance psychosocial wellbeing. Furthermore, there have been tragic instances of COVID-19 patients dying alone without their loved ones nearby. These risks can be mitigated to some extent by rapid testing of vulnerable population caregivers and having good, honest, and in-time communications with families early and often (18).

With COVID-19 spreading widely, particularly in larger metropolitan areas within the United States, overwhelming numbers of older adults with comorbidities and frailty have led to a high number of older adults requiring medical attention, including ICU care, at the same time. Several of these patients have required intubation and prolonged mechanical ventilation. This has led to a shortage of ventilators, compelling frontline clinicians to make difficult and uncomfortable choices in rationing life-sustaining care. With no definitive therapy available, providers have little to offer. In these situations, discussions with palliative care teams are critical to decide the goals of care and to communicate with families who are unable to visit their loved ones. These teams generally consist of a palliative care physician, nurse practitioner or physician assistant, social worker, and nurse case manager. These teams have been crucial in helping frontline clinicians draft management plans, decide on goals of care, and communicate with families (19). 
Table 1. COVID-19 response plan components for nursing homes

COVID-19 response plan components

Rapid identification and management of ill residents

Optimizing supplies and resources

Sick leave policies and other occupational health considerations

Education and training

Surge capacity for staffing, equipment and supplies, and postmortem care

\section{Comments}

- Temperature check and symptom screen at least once daily

- Immediate testing of symptomatic residents

- Rapid implementation of appropriate transmission-based precautions

- Determine threshold for active surveillance (testing all residents), if available, to detect asymptomatic carriers

- Strict limitation of visitors

- Symptom screen (and consider temperature check) for all staff daily upon arrival

- Anticipate PPE, hand sanitizer, and environmental cleaning supply needs

- Anticipate potential supply chain interruptions

- Use PPE burn calculators

- Partner with regional institutions and health department for resources

- Anticipate staffing shortages

- Consider hazard pay

- Normalize sick leave/discourage presenteeism (working while ill)

- Focus on hand hygiene, PPE donning and doffing, and recognition of COVID-19 signs and symptoms

- Open and frequent communication with health department, local hospitals, families, patients and staff to foster trust and collaboration

- Consider plan for managing COVID-19 cases including:

unit and staff cohorting

attention to and monitoring nutrition, function, and psychosocial health using technology communication with health department

advanced care planning and end-of-life care

postmortem infection control plan

As a result of consolidation of outpatient operations and social distancing measures, healthy community-dwelling older adults are often devoid of necessary support, knowledge, and adaptive strategies to manage their day-to-day routines and are at risk of worsening their underlying comorbidities. Communicating with "well and worried" older adults using virtual lectures via senior community-based centers and learning communities, and ramping up virtual and video health visits have helped mitigate some of these concerns - albeit only for those who have access to these resources (20).

\section{Public health and policy impact}

Our health care system has evolved tremendously in the last decade - largely in response to reducing inpatient hospital stays. Innovative models such as using nursing facilities for short-stay rehabilitation, longterm acute care hospitals for patients requiring long-term ventilation, house calls, and hospital-at-home programs to allow seniors to "age-in-place," have moved health care out of the hospital walls, and helped older adults recover from acute illness and maintain their functional independence. However, this pandemic has shown glaring gaps in preparing these sectors and patients for emergencies, particularly those related to preventing infections and managing outbreaks.

Developing, adopting, and testing disaster preparedness plans, including for pandemics, in post-acute care settings including nursing homes, assisted living facilities, and long-term acute care hospitals is of paramount importance, given the wide spectrum of COVID-19 symptoms and high risk of severe outcomes in older adults. This can be particularly challenging considering the limited human and material resources dedicated to infection prevention at many facilities. Much like the rest of the country, public health and infection control infrastructure in these facilities needs to be substantially enhanced and modernized.

In general, the United States has become too reliant on single-use products, including standard personal protective equipment (PPE). With resources generally directed to acute care hospitals first, these challenges are compounded in poorly resourced facilities that care for an aging demographic. Increased use of items that can be disinfected and reused can allow for a much safer and controlled response to outbreaks. This pandemic has taught us that the supply chain for medical and infection prevention supplies needs geographic diversification. A paucity of manufacturing sources and just-in-time inventory management limit the ability 
to rapidly increase PPE supplies in times of high demand. Having stockpiles of important infection prevention items, such as N95 and surgical masks, can provide much needed protection for health care workers while a health care institution determines how to replenish supplies. There is also a need for more no-touch technology in hospitals and other care sites to decrease transmission risk for health care workers, patients, and visitors. The situation is often worse in nursing homes, where typically, less PPE is available on site, and adaption of novel infection prevention technology often occurs much later than in acute care settings.

A clear lesson from the COVID-19 pandemic is that nursing homes are an important, high-risk target for emerging pathogens due to the presence of vulnerable patients, frequent sharing of medical staff and patients with multiple other institutions, and frequent visitors (14). Proactive pandemic planning in nursing homes includes active symptom screening for illness among health care workers and staff, including temperature checks, strict visitor limitations, limiting group activities, and practicing universal masking and droplet precautions once local community transmission of COVID-19 has been identified (Table 1) (21). Developing a robust pandemic response plan in nursing homes requires not only physical but human capital. Sustaining a healthy and well-trained workforce is essential and requires investment through standardized education on hand hygiene and PPE use, as well as generous sick leave policies and overall prioritization of staff retention. Established lines of communication between institutional medical directors, corporate leadership, and local health department authorities allow for rapid coordination of infection control response plans.

COVID-19 has shown the ability to devastate vulnerable populations, including older adults. It has also resulted in rapid and unprecedented changes in health care delivery and restrictions on typical day-to-day community activities, both of which have an outsized impact on older adults. Public policy goals should prioritize pandemic preparedness in nursing homes, as well as civic and local government-based support programs for community-dwelling older adults, to ensure that risk of infection is mitigated while promoting wellness during a period of stress and uncertainty.

Address correspondence to: John P Mills, University of Michigan Medical School, Division of Infectious Diseases, University Hospital South Room F4177, Ann Arbor, Michigan 48109, USA. Phone: 734.998.6060; Email: millsjo@umich.edu. Or to: Lona Mody, University of Michigan Medical School, Division of Geriatric and Palliative Care Medicine, 300 N. Ingalls Road, Room 905, Ann Arbor, Michigan 48109, USA. Phone: 734.764.8942; Email: lonamody@umich.edu.

1. Andersen KG, Rambaut A, Lipkin WI, Holmes EC, Garry RF. The proximal origin of SARS-CoV-2. Nat Med. 2020;26(4):450-452.

2. [No authors listed]. COVID-19 Case Tracker. Johns Hopkins University and Medicine Coronavirus Resource Center. https://coronavirus.jhu.edu/. Accessed May 1, 2020.

3. Wu Z, McGoogan JM. Characteristics of and important lessons from the coronavirus disease 2019 (COVID-19) outbreak in China: Summary of a report of 72314 cases from the Chinese Center for Disease Control and Prevention. JAMA. 2020;323(13):1239-1242.

4. CDC COVID-19 Response Team. Preliminary estimates of the prevalence of selected underlying health conditions among patients with coronavirus disease 2019 - United States, February 12-March 28, 2020. MMWR Morb Mortal Wkly Rep. 2020;69(13):382-386.

5. Guan WJ, et al. Clinical characteristics of coronavirus disease 2019 in China. N Engl J Med. 2020;382(18):1708-1720.

6. Dudley JP, Lee NT. Disparities in age-specific morbidity and mortality from SARS-CoV-2 in China and the Republic of Korea. Clin Infect Dis. 2020:ciaa354.

7. Grasselli G, et al. Baseline characteristics and outcomes of 1591 patients infected with SARS-CoV-2 admitted to ICUs of the Lombardy Region, Italy. JAMA. 2020;323(16):1574-1581

8. Lian J, et al. Analysis of epidemiological and clinical features in older patients with corona virus disease 2019 (COVID-19) out of Wuhan. Clin Infect Dis. 2020:ciaa242.

9. Wang D, et al. Clinical characteristics of 138 hospitalized patients with 2019 novel coronavirus-infected pneumonia in Wuhan, China. JAMA. 2020;323(11):1061-1069.

10. Zhou F, et al. Clinical course and risk factors for mortality of adult inpatients with COVID-19 in Wuhan, China: a retrospective cohort study. Lancet. 2020;395(10229):1054-1062.

11. Kimball A, et al. Asymptomatic and presymptomatic SARS-CoV-2 infections in residents of a long-term care skilled nursing facility - King County, Washington, March 2020. MMWR Morb Mortal Wkly Rep. 2020;69(13):377-381.

12. Lauer SA, et al. The incubation period of coronavirus disease 2019 (COVID-19) from publicly reported confirmed cases: estimation and application [published ahead of print March 10, 2020]. Ann Intern Med. https://doi.org/10.7326/M20-0504.

13. Klok FA, et al. Incidence of thrombotic complications in critically ill ICU patients with COVID-19 [published ahead of print April 10, 2020]. Thromb Res. https://doi.org/10.1016/j.thromres.2020.04.013.

14. McMichael TM, et al. Epidemiology of COVID-19 in a long-term care facility in King County, Washington [published ahead of print March 10, 2020]. N Engl J Med. https://doi.org/10.1056/NEJMoa2005412.

15. Bhimraj A, et al. Infectious Disease Society of America guidelines on the treatment and management of patients with COVID-19. 
Infectious Diseases Society of America. https://www.idsociety.org/COVID19guidelines. Published April 11, 2020. Accessed May $1,2020$.

16. Fang L, Karakiulakis G, Roth M. Are patients with hypertension diabetes mellitus at increased risk for COVID-19 infection? Lancet Respir Med. 2020;8(4):E21.

17. [No authors listed]. Patients taking ACE-i and ARBs who contract COVID-19 should continue treatment, unless otherwise advised by their physician. American Heart Association. https://newsroom.heart.org/news/patients-taking-ace-i-and-arbswho-contract-covid-19-should-continue-treatment-unless-otherwise-advised-by-their-physician. Published March 17, 2020. Accessed May 1, 2020.

18. Grabowski DC, Joynt Maddox KE. Postacute care preparedness for COVID-19: thinking ahead [published ahead of print March 25, 2020]. JAMA. https://doi.org/10.1001/jama.2020.4686.

19. Emanuel EJ, et al. Fair allocation of scarce medical resources in the time of COVID-19 [published ahead of print March 23 , 2020]. N Engl J Med. https://doi.org/10.1056/NEJMsb2005114.

20. Hansen RJ, Talmage CA, Thaxton SP, Knopf RC. Barriers to age-friendly universities (AFU): Lessons from Osher Lifelong Learning Institute demographics and perceptions. Gerontol Geriatr Educ. 2019;40(2):221-243.

21. [No authors listed]. Preparedness checklist for nursing homes and other long-term care settings. Centers for Disease Control and Prevention. https://www.cdc.gov/coronavirus/2019-ncov/hcp/long-term-care-checklist.html. Accessed May 1, 2020 